\title{
Confocal microscopic analysis of optical crosstalk in GaN micro-pixel light-emitting diodes
}

\author{
K. H. Li, Y. F. Cheung, W. S. Cheung, and H. W. Choi ${ }^{\text {a) }}$ \\ Department of Electrical and Electronic Engineering, The University of Hong Kong, Hong Kong, Hong Kong
}

(Received 28 July 2015; accepted 18 October 2015; published online 27 October 2015)

\begin{abstract}
The optical crosstalk phenomenon in GaN micro-pixel light-emitting diodes (LED) has been investigated by confocal microscopy. Depth-resolved confocal emission images indicate light channeling along the GaN and sapphire layers as the source of crosstalk. Thin-film micro-pixel devices are proposed, whereby the light-trapping sapphire layers are removed by laser lift-off. Optical crosstalk is significantly reduced but not eliminated due to the remaining GaN layer. Another design involving micro-pixels which are completely isolated is further proposed; such devices exhibited low-noise and enhanced optical performances, which are important attributes for high-density micro-pixel LED applications including micro-displays and multi-channel optical communications. () 2015 AIP Publishing LLC. [http://dx.doi.org/10.1063/1.4934840]
\end{abstract}

Micro-pixelation transforms planar light-emitting diodes (LEDs) into more efficient and functional emitters. Efficiency is enhanced by virtue of improved light extraction efficiency, releasing light which would otherwise have been confined within the rectangular cubic cavities by integrating regularly ordered three-dimensional patterns onto the emission surface(s),${ }^{1-3}$ which are typically formed by etching through a masking layer beyond the active region. Apart from electrically inter-connecting all pixels to function as a homogeneous emitter, they can also be wired up for individual, matrix, or group addressing. Two-dimensional arrays based on the III-nitride materials emitting at ultraviolet and visible wavelengths have found uses as emissive micro-displays, ${ }^{4}$ sources for maskless photolithography, ${ }^{5}$ and structured-light microscopy, ${ }^{6}$ as well as emitters for optical communications ${ }^{7}$ amongst many others. While the enhancement of light extraction efficiency is adequately achieved through micro-structuring, the optical performances of addressable arrays are less satisfactory due to noticeable optical crosstalk. When a single pixel illuminates, adjacent pixels and regions appear illuminated simultaneously. The cause of optical crosstalk was initially thought to be due to emission from the pixel sidewalls; this is subsequently found not to be the case in this work as the coverage of sidewalls with an opaque coating did not alleviate the problem. The seriousness of optical crosstalk can be perceived from the example of multi-channel optical communications using micro-pixelated LED arrays as sources. When a light pulse is sent down an optical channel from a single emitter, adjacent channels may be carrying the same light pulse due to optical crosstalk, resulting in less-than-optimal system performance. The development of red-green-blue (RGB) micro-pixelated LED has also hampered by optical crosstalk. This proposed color-tunable device consists of group-addressable micropixels which emit RGB colors by selective coating of the pixels with a suitable color converter. ${ }^{8}$ Due to optical crosstalk, when a group of pixels emitting a single color is

\footnotetext{
${ }^{\text {a) }}$ Author to whom correspondence should be addressed. Electronic mail: hwchoi@hku.hk. Tel.: (852) 28592693. Fax: (852) 25598738.
}

turned on, the adjacent differently colored pixels would also appear to be emitting simultaneously, disrupting its designed functionality.

Addressing the crosstalk issue is pertinent for further development of III-nitride micro-emitter arrays to compete with existing display technologies based on liquid-crystals and organic LEDs (OLEDs), especially in view of the multiple advantages of the nitride-based emitters in terms of quantum efficiencies, temporal response, reliabilities, and robustness. Liquid-crystal displays (LCDs), being backlightilluminated, are not spared from optical crosstalk effects. As the LCD optical filters cannot be completely opaque, finite amounts of light will always be emitted from every pixel so that a true black output cannot be realistically achieved, compromising on image contrast as displays or reduced signal-to-noise ratio in optical communication systems, not to mention their slower temporal responses. ${ }^{9}$ On the other hand, emissive OLED displays do not rely on backlights and theoretically should not suffer from optical crosstalk effects. However, OLEDs currently suffer from limited lifespans, susceptibility to water damages and low manufacturing yields; none of these apply to the GaN-based emitters. In fact, blue OLEDs typically have lifetimes of a few thousand hours compared to tens of thousands of hours for the GaN counterparts. If only the issue of optical crosstalk be resolved, the future of GaN-based displays would be bright.

Figures 1(a) and 1(b) illustrate microphotographs of RGB pixels from typical LCD and OLED displays, while the pixels from a monochromatic III-nitride micro-pixel LED are shown in Fig. 1(c), which evidently are emitting amidst strong background emission despite not having a backlight. Such crosstalk effects could arise from the architecture of the emitter (which is the subject of this study) or emission divergence. To eliminate the effects caused by divergence, the same images are captured again using a confocal microscope, which allows light from out-of-focus planes to be filtered. Confocal images of the displays or devices in Figs. 1(a)-1(c) captured from the top planes are shown in Figs. 1(d)-1(f), respectively; the data are acquired under identical measurement settings, including detector gain, exposure 

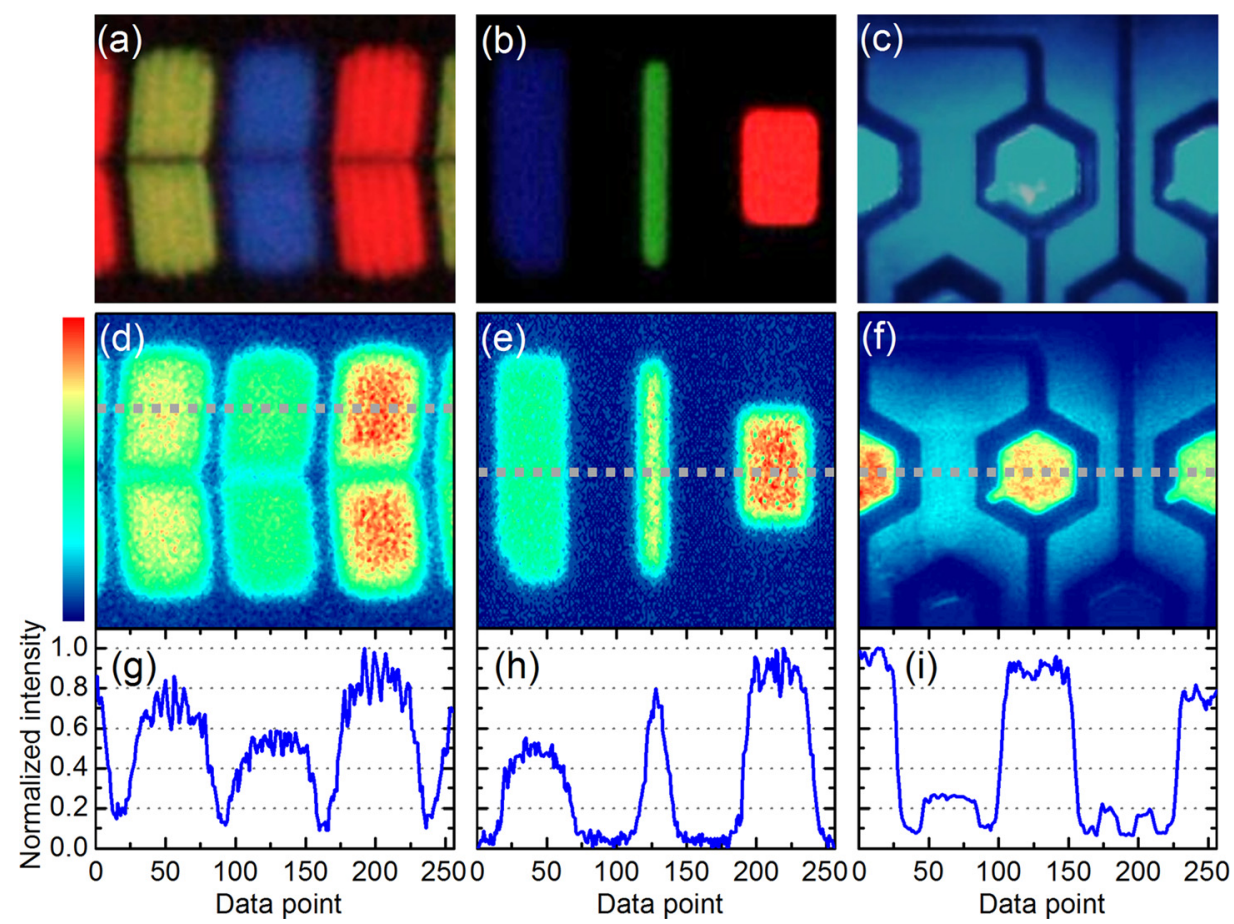

FIG. 1. Microphotographs of RGB pixels from (a) LCD and (b) OLED displays, and (c) monochromactic III-nitride micro-pixel LEDs. Planar intensity maps of (d) LCD, (e) OLED, and (f) III-nitride pixels captured by confocal microscopy. (g)-(i) Plots of emission intensities along the dotted lines in Figs. 1(d)-1(f), respectively. time, and scanning speed. Emission intensity profiles along the dotted lines shown in Figs. 1(d)-1(f) are plotted in Figs. 1(g)-1(i), respectively. As the emitters of different technologies are operated at unequal currents and emit with nonidentical luminous intensities, the extent of optical crosstalk can only be fairly compared by evaluating a signal-to-noise $(\mathrm{S} / \mathrm{N})$ figure from the ratio of maximum signal intensity (signal within a pixel) to maximum noise intensity (signal beyond a pixel). At an $\mathrm{S} / \mathrm{N}$ ratio of $\sim 3.7$, the micro-pixel LED is found to be inferior to the LCD and OLED with S/N ratios of $\sim 4.4$ and $\sim 13.3$.

Having quantitatively established the severity of the optical crosstalk effects in the micro-pixel LEDs, it is imperative to develop strategies to overcome them. Variants of micro-pixel LED structures have also been reported, including flip-chip devices having pixels on the upward-facing n-GaN surfaces, ${ }^{10}$ planar devices with plasma-treated pixel isolation regions, ${ }^{11}$ as well as geometrically shaped pixels. ${ }^{12}$ Although each of these designs achieves its own specific target, none of them addresses the optical crosstalk issue. Complete optical isolation between pixels can be achieved by using discrete LEDs devices for each pixel, which is the strategy of choice employed for the assembly of RGB fullcolor display panels. ${ }^{13}$ Given the dimensions of individual chips and the sizes of the arrays, conventional pick-and-place die bonding does not offer the required precision for the task. To overcome these limitations, technologies based on wafer-scale transfer printing have been developed for manufacturing of RGB microdisplays. ${ }^{14-16}$ In this study, confocal microscopy is employed to investigate the optical crosstalk phenomenon from GaN micro-pixel LED devices, with the objective of understanding the mechanism of optical crosstalk in these devices, as well as for the development of solutions to overcome the problem. The proposed solution comes in the form of thin-film micro-pixel LEDs whereby the sapphire substrate, a major source of light recycling, is removed by laser lift-off (LLO) processes so that optical crosstalk is mitigated.

The micro-pixel devices in this study are fabricated on metal-organic chemical vapor deposition (MOCVD)-grown epitaxial wafers on 2-in. c-plane sapphire substrates. The epi-structure consists of $3 \mu \mathrm{m}$ of undoped $\mathrm{GaN}, 3 \mu \mathrm{m}$ of $n$ doped GaN, 10 pairs of $\mathrm{InGaN} / \mathrm{GaN}(3 \mathrm{~nm} / 12 \mathrm{~nm})$ quantum wells, and $150 \mathrm{~nm} p$-doped $\mathrm{GaN}$. The fabrication begins with the deposition of a thin 200-nm indium-tin-oxide (ITO) current spreading layer onto $p$-GaN. The hexagonal-shaped pixel array with pixel dimensions of $\sim 60 \mu \mathrm{m}$ is photolithographically defined, and the unmasked region is dryetched by $\mathrm{Cl}_{2}$-based plasma down to the $n$-GaN layer. Prior to metal deposition, a $50 \mathrm{~nm} \mathrm{SiO}_{2}$ electrically insulating film is evaporated, followed by the exposure of the contact areas by wet etching through the oxide layer via another photolithographically defined mask. The $p$ - and $n$-contacts are then e-beam deposited and annealed, completing the fabrication of the micro-pixel LED ( $\mu$ LED). The sidewalls of the micro-pixels are designed to be fully covered by metallic interconnects. The thin-film micro-pixel LEDs ( $t f-\mu \mathrm{LEDs})$ are processed from the $\mu$ LEDs via a double-flip process in order to transfer the fabricated device from its original sapphire substrate to a copper sub-mount. The $p$-face of the device is temporarily attached to a glass substrate, followed by detachment of the sapphire substrate by LLO using the collimated beam from a $266 \mathrm{~nm} \mathrm{Nd}$ :YAG laser (Continuum Surelite). After bonding onto a copper sub-mount, the glass substrate is removed. The micro-pixel LED with completely isolated pixels (iso- $\mu \mathrm{LED}$ ) is fabricated using a different fabrication process flow altogether, which begins with the coating of a bi-layer of $\mathrm{Ni} / \mathrm{Au}(40 / 200 \mathrm{~nm})$ onto the $p$-face of the same starting LED wafer. The metal-coated surface is waferbonded to a Si substrate followed by removal of the sapphire substrate by LLO. The film is then thinned down to $\sim 2 \mu \mathrm{m}$ by $\mathrm{BCl}_{3}$-based inductively coupled plasma (ICP) etching 
down to the $n$-GaN layer. The same pixel array is then formed on this thin-film by etching through all the GaN layers down to the metal layer. Following oxide isolation, metallic interconnects are deposited. Cross-sectional schematic diagrams of the $\mu \mathrm{LED}, t f-\mu \mathrm{LED}$, and $i s o-\mu \mathrm{LED}$ are depicted in Figs. 2(a)-2(c), while Figs. 2(d)-2(f) illustrate planar views of the respective fabricated devices operated at $9 \mathrm{~mA}$. The confocal microscopy images are obtained using a Carl Zeiss LSM700 laser-scanning confocal microscope in the light collection mode; the laser is not turned on during operation. Using a $5 \times$ objective with a numerical aperture (NA) of 0.13 in combination with a pinhole size of 0.3 airy unit, a depth of field (axial resolution) of $\sim 8.6 \mu \mathrm{m}$ is achieved. Each 2-D planar micrographs represents a single optical slice consisting of $256 \times 256$ data points, while the cross-sectional images are constructed by $z$-stacking 100 confocal slices in steps of $10 \mu \mathrm{m}$.

As previously established, emission from a $\mu \mathrm{LED}$ is characterized by strong background emission as if a backlight is present, as evident from Fig. 1(a) as well as Fig. 2(d). In order to discover the cause of the background emission, it is necessary to probe the distribution of light within the device structure, a task which can be fulfilled by confocal microscopy. A confocal $z$-stack scan is collected from an illuminated $\mu \mathrm{LED}$ device, from which a cross-sectional emission profile can be generated as depicted in Fig. 2(g). The $z$-scanning range spans from the base of the sapphire substrate to $0.6 \mathrm{~mm}$ above the surface of the device, enabling detection of light both within and outwith the LED structure. The position of its active region is indicated with a white dashed line in the figure. Suppression of sidewall emission by the annular ring contact is clearly evident from the presence of a dark region adjacent to the edge of the micro-pixel. Above the active region, the light gets extracted from the pixel through a metal frame aperture and diverges into an emission cone. Beneath the active region, a massive amount of light is also detected, the amount of which is no less than those emitted upwards. This corresponds to the portion of light which emits downwards from the active region, as well as they light rays which have not been extracted and thus are reflected downwards due to total internal reflections (TIRs). If a ray is reflected due to TIR then it is likely also to be
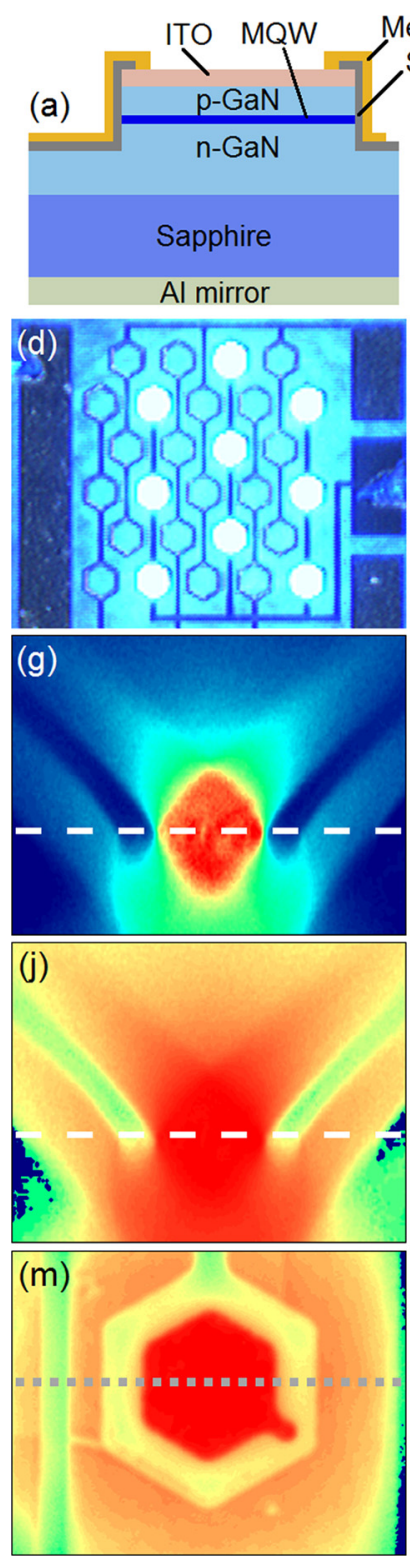
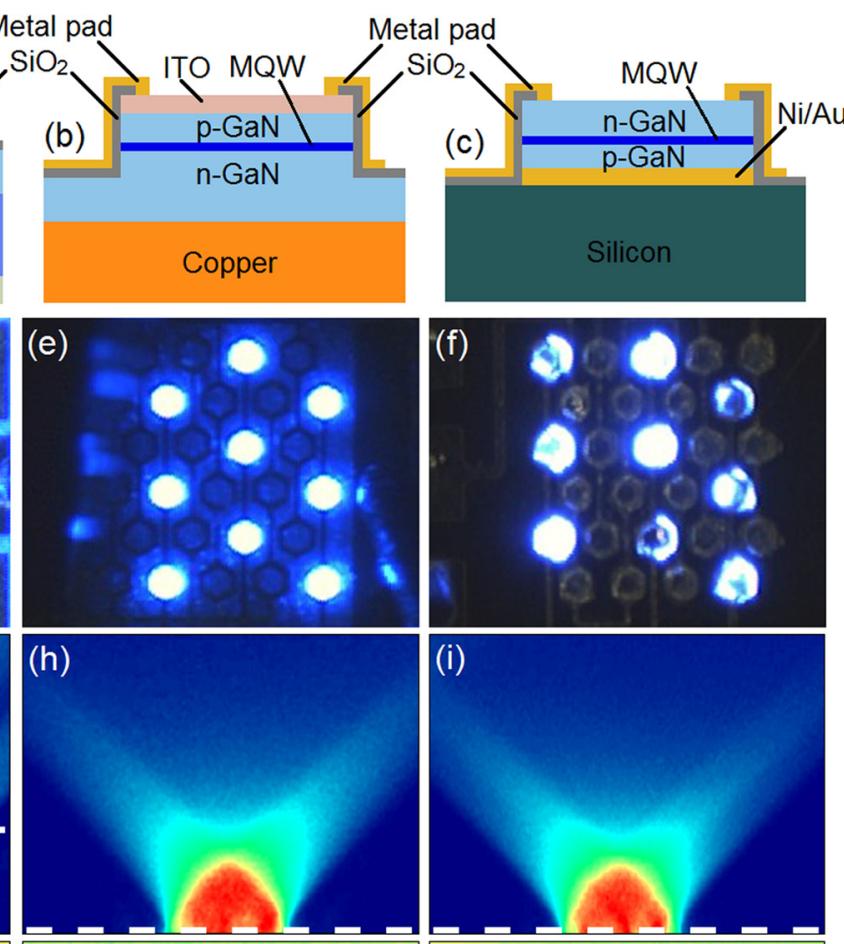

(k)

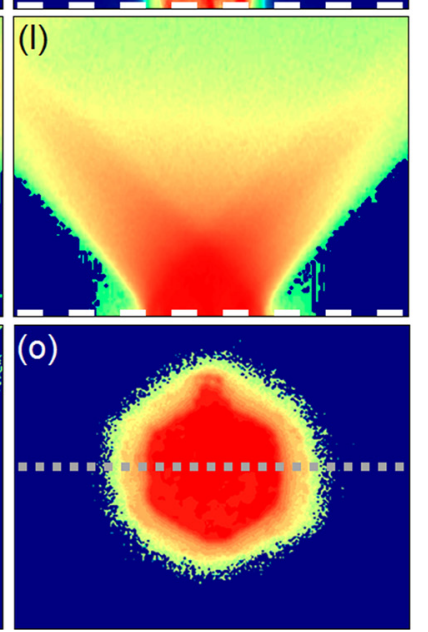

(c)

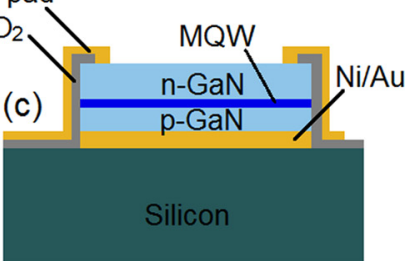

FIG. 2. (a) Cross-sectional schematic diagrams depicting architectures of (a) $\mu \mathrm{LED}$, (b) $t f-\mu \mathrm{LED}$, and (c) iso- $\mu \mathrm{LED}$. Microphotographs showing emission from a group of pixels of the respective devices in (d)-(f). Cross-sectional emission intensity maps $\left(142 \times 1000 \mu \mathrm{m}^{2}\right.$ along $\mathrm{x}-\mathrm{z}$ plane) of the respective devices plotted on linear scale (g)-(i) and logarithmic scale (j)-(l). Planar intensity maps of the respective devices plotted on logarithmic scale (m)-(o). 

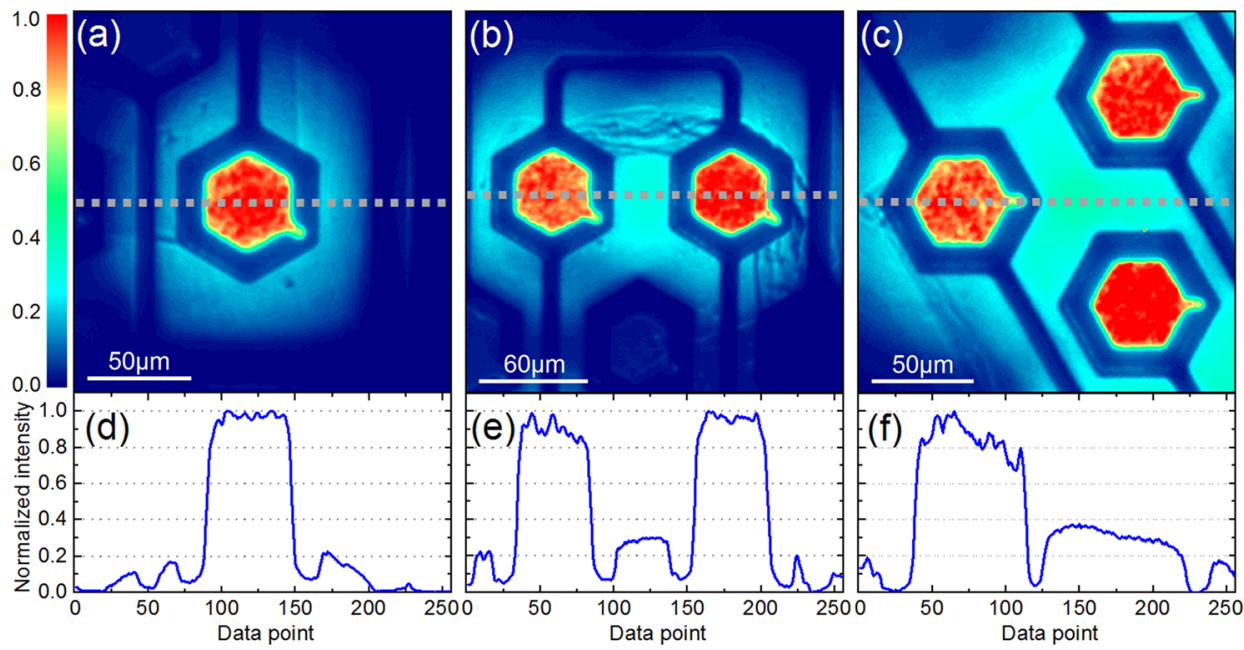

FIG. 3. Confocal images of emission from (a) single, (b) double, and (c) triple pixels in a $\mu$ LED. (d)-(f) Plots of emission intensity profiles along the dotted lines (a)-(c), respectively. reflected along parallel and perpendicular interfaces as the conditions for TIR will continue to be fulfilled. These confined light rays will thus channel along the planar GaN and sapphire layers until escaping from the surrounding surfaces due to surface roughnesses.

The confocal images in Figs. 3(a)-3(c) provide further evidence on the optical channeling effects, whereby single, double, and triple pixels in an array (each pixel driven at the current of $1 \mathrm{~mA}$ ) are illuminated. Plots of emission intensity profiles along the dotted line segments shown are plotted beneath the respective confocal images, from which $\mathrm{S} / \mathrm{N}$ ratios are evaluated as $4.5,3.3$, and 2.7 , respectively. It is clearly observed that the $\mathrm{S} / \mathrm{N}$ ratio drops as the number of illuminated pixels increases, supporting the postulation of the optical channeling effect.

Having identified the source of optical crosstalk micropixel LEDs, the design of the device architecture can be modified accordingly to overcome the problem. As optical channeling occurs in the GaN and sapphire layer, ${ }^{17}$ and that the thickness of the sapphire substrate is significantly thicker than the GaN epilayer, it should be adequate to remove the sapphire substrate from the devices. Incidentally, sapphire removal by LLO is an established process for fabricating thin-film GaN LEDs, which has been developed primarily for improving heat-sinking in high-powers LEDs due to the low thermal conductivity of sapphire. ${ }^{18}$ The same technique can now be employed for the fabrication of thin-film micropixel LEDs, the process flow of which is described in the section on experimental details, and a cross-section schematic diagram of the thin-film version of the same micropixel LED is shown in Fig. 2(b), while Fig. 2(e) shows a microphotograph of the illuminated devices. Compared with the conventional micro-pixel LED in Fig. 2(d), a marked reduction of the background signal is observed. Crosssection emission profile of the $t f$ - $\mu \mathrm{LED}$, obtained from the confocal $z$-stacks, is plotted in Fig. 2(h). Compared to the conventional $\mu \mathrm{LED}$, the massive amount of light trapped beneath the pixel is no longer observed since the lighttrapping sapphire layer has been removed, and the light rays are instead reflected by the metallic mirror at the interface. Of course, upon close observation of the microphotograph of Fig. 2(e), one can see that although the background signal has been significantly suppressed, it is by no means eliminated. To achieve the highest possible contrast, the elimination of the background emission is indispensable; this is only possible if the individual pixels are completely isolated. In other words, the GaN layer beneath the pixels linking the pixels that give rise to optical channeling must be eliminated. To fabricate such devices, the process flow must be further modified. Instead of fabricating the LED on a GaN-on-sapphire wafer, the fabrication has to be carried out on a GaN thin-film wafer, details of which are described in the earlier in experimental details paragraph. Cross-section schematic diagram of an iso- $\mu \mathrm{LED}$ is depicted in Fig. 2(c), while a microphotograph of the fabricated device is shown in Fig. 2(f). Clearly, emission is only detected from within the micro-pixels; the background appears to be completely dark. The cross-sectional emission profile obtained from the confocal $z$-stack of the $i s o-\mu \mathrm{LED}$ in Fig. 2(i) appears identical to that of the $t f$ - $\mu \mathrm{LED}$, since background emission from the $t f-\mu \mathrm{LED}$ is sufficiently weak. Their differences become visible when the same set of data is plotted on a logarithmic scale in Figs. 2(j)-2(1), revealing slightly more emission at the edges of the micro-pixels. The extent of crosstalk can also be quantified by evaluating the $\mathrm{S} / \mathrm{N}$ ratios from the emission intensity profiles of single pixels from the $\mu \mathrm{LED}, t f$ $\mu \mathrm{LED}$, and iso- $\mu \mathrm{LED}$, respectively, plotted in Fig. 4(a), which have been generated from their respective planar confocal images in Figs. 2(m)-2(o). The $t f$ - $\mu$ LED plot exhibits two weak noise peaks beyond the micro-pixel, giving a large $\mathrm{S} / \mathrm{N}$ ratio of $\sim 15.9$, while insignificant noise intensities from the $i$ so- $\mu \mathrm{LED}$ contributes to a huge $\mathrm{S} / \mathrm{N}$ ratio exceeding 100 ,
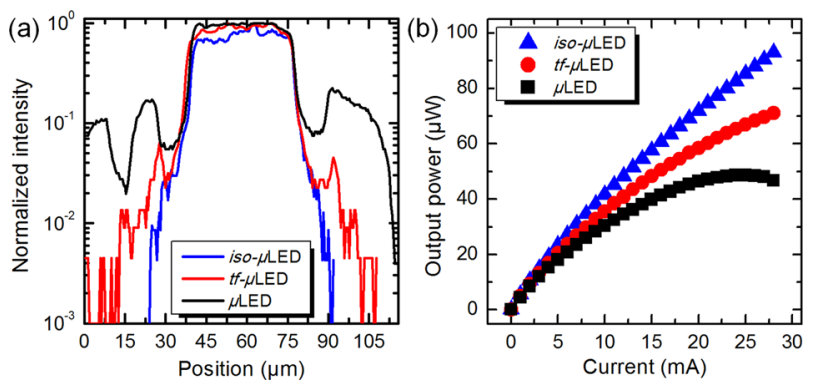

FIG. 4. (a) Plots of emission intensity profiles along the dotted lines in Figs. 2(m)-2(o), respectively. (b) Intensity-current (L-I) characteristics of micropixel LEDs. 
confirming the perfectly dark background of the iso- $\mu \mathrm{LED}$ and testifying to the effectiveness of the design.

Since the reduction/elimination of optical crosstalk in $t f$ $\mu \mathrm{LED}$ and iso- $\mu \mathrm{LED}$ is achieved by the suppression of laterally guided light, improvement of light extraction is also implied. To verify this, the emitted light from a single pixel in each of the three micro-pixel LEDs is collected with a $20 \times$ objective with NA of 0.42 , which is then sensed by a Si-photodiode. Figure 4(b) shows plots of measured optical powers as functions of injection current under DC conditions. Compared with the $\mu \mathrm{LED}$, the $t f-\mu \mathrm{LED}$, and iso- $\mu \mathrm{LED}$ emit $\sim 14 \%$ and $\sim 30 \%$ more light at a low injection current of $5 \mathrm{~mA}$. The absence of an absorbing ITO, layer as well as complete elimination of light trapping, accounts for the higher optical output from the $i s o-\mu$ LED over the $t f-\mu$ LED. At a higher current of $28 \mathrm{~mA}$, the corresponding enhancement factors are further increased to $\sim 50 \%$ and $\sim 100 \%$, which are readily attributed to the superior heat-sinking capabilities of the thin-film devices. With unparalleled optical performance over conventional micro-pixel LED architectures, the iso- $\mu \mathrm{LED}$ is an ideal platform for the development of micro-displays with performances to rival existing technologies.

In summary, the cause of optical crosstalk from GaN micro-pixel LEDs has been investigated by depth-resolved confocal microscopy, and identified to be optical channeling in the transparent layers beneath the active regions. Using LLO processes, the sapphire substrate is removed to form thin-film micro-pixel LEDs, whose optical crosstalk performances are significantly improved. To achieve the best possible contrast, devices with optically isolated micropixels have also been developed; negligible optical crosstalk is achieved with this design. These devices are also capable of emitting more light at the same current due to minimized light trapping as well as better heat-sinking. GaN microdisplays, parallel emitters, and color-tunable devices will benefit from this low-noise and high-efficiency micro-pixel LED architecture.
This work was supported by the Theme-based Research Scheme (T23-612/12-R) of the Research Grant Council of Hong Kong.

${ }^{1}$ S. X. Jin, J. Li, J. Z. Li, J. Y. Lin, and H. X. Jiang, Appl. Phys. Lett. 76(5), 631 (2000).

${ }^{2}$ H. W. Choi, C. W. Jeon, M. D. Dawson, P. R. Edwards, R. W. Martin, and S. Tripathy, J. Appl. Phys. 93(10), 5978 (2003).

${ }^{3}$ S. X. Jin, J. Shakya, J. Y. Lin, and H. X. Jiang, Appl. Phys. Lett. 78(22), 3532 (2001).

${ }^{4}$ H. W. Choi, C. W. Jeon, and M. D. Dawson, IEEE Electron Device Lett. 25(5), 277 (2004).

${ }^{5}$ D. Elfstrom, B. Guilhabert, J. McKendry, S. Poland, Z. Gong, D. Massoubre, E. Richardson, B. R. Rae, G. Valentine, G. Blanco-Gomez, E. Gu, J. M. Cooper, R. K. Henderson, and M. D. Dawson, Opt. Express 17(26), 23522 (2009).

${ }^{6}$ V. Poher, H. X. Zhang, G. T. Kennedy, C. Griffin, S. Oddos, E. Gu, D. S. Elson, J. M. Girkin, P. M. W. French, M. D. Dawson, and M. A. A. Neil, Opt. Express 15(18), 11196 (2007).

${ }^{7}$ J. J. D. McKendry, D. Massoubre, S. L. Zhang, B. R. Rae, R. P. Green, E. Gu, R. K. Henderson, A. E. Kelly, and M. D. Dawson, J. Lightwave Technol. 30(1), 61 (2012).

${ }^{8}$ W. N. Ng, C. H. Leung, P. T. Lai, and H. W. Choi, Phys. Status Solidi C 5, 2198 (2008).

${ }^{9}$ T. Tsujimura, OLED Display Fundamentals and Applications (John Wiley \& Sons, 2012).

${ }^{10}$ Z. Gong, E. Gu, S. R. Jin, D. Massoubre, B. Guilhabert, H. X. Zhang, M. D. Dawson, V. Poher, G. T. Kennedy, P. M. W. French, and M. A. A. Neil, J. Phys. D: Appl. Phys. 41, 094002 (2008).

${ }^{11}$ D. Massoubre, E. Y. Xie, B. Guilhabert, J. Herrnsdorf, E. D. Gu, I. M. Watson, and M. D. Dawson, Semicond. Sci. Technol. 29(1), 015005 (2014).

${ }^{12}$ P. P. Maskant, H. Shams, M. Akhter, W. Henry, M. J. Kappers, D. D. Zhu, C. J. Humphreys, and B. Corbett, Appl. Phys. Express 6(2), 022102 (2013).

${ }^{13}$ K. McGroddy, H.-H. Hu, A. Bibl, C. K. T. Chan, and D. A. Haeger, U.S. patent application 14/194,509 (2015).

${ }^{14}$ A. Carlson, A. M. Bowen, Y. G. Huang, R. G. Nuzzo, and J. A. Rogers, Adv. Mater. 24(39), 5284 (2012).

${ }^{15}$ T. I. Kim, Y. H. Jung, J. Z. Song, D. Kim, Y. H. Li, H. S. Kim, I. S. Song, J. J. Wierer, H. A. Pao, Y. G. Huang, and J. A. Rogers, Small 8(11), 1643 (2012).

${ }^{16}$ J. Yoon, S.-M. Lee, D. Kang, M. A. Meitl, C. A. Bower, and J. A. Rogers, Adv. Opt. Mater. 3, 1313 (2015).

${ }^{17}$ A. David, T. Fujii, R. Sharma, K. McGroddy, S. Nakamura, S. P. DenBaars, E. L. Hu, C. Weisbuch, and H. Benisty, Appl. Phys. Lett. 88, 061124 (2006).

${ }^{18}$ K. H. Li, Y. F. Cheung, Q. Zhang, and H. W. Choi, IEEE Photonics Technol. Lett. 25(4), 374 (2013). 